viral phenotypes. J. Leukoc. Biol. 80:965-972.

24. Newrzela, S., et al. 2008. Resistance of mature T cells to oncogene transformation. Blood. 112:2278-2286.

25. Costin, J.M. 2007. Cytopathic mechansims of HIV-1. Virology. 4:1-22.

26. Rossi, J.J., June, C.H., and Kohn, D.B. 2007. Genetic therapies against HIV. Nat. Biotechnol. 25:1444-1454.
27. Montini, E., et al. 2006. Hematopoietic stem cell gene transfer in a tumor-prone mouse model uncovers low genotoxicity of lentiviral vector integration. Nat. Biotechnol. 24:687-696.

28. Modlich, U., et al. 2008. Leukemia induction after a single retroviral vector insertion in Evi1 or Prdm16. Leukemia. 22:1519-1528.
29. Nienhuis, A.W., Dunbar, C.E., and Sorrentino, B.P. 2006. Genotoxicity of retroviral integration in hematopoietic cells. Mol. Ther. 13:1031-1049.

30. Dupuy, A.J., Akagi, K., Largaespada, D.A., Copeland, N.G., and Jenkins, N.A. 2005. Mammalian mutagenesis using a highly mobile somatic Sleeping Beauty transposon system. Nature. 436:221-226.

\title{
Wnt therapy for bone loss: golden goose or Trojan horse?
}

\author{
Greg $\mathrm{H}$. Enders
}

Department of Medicine, Epigenetics and Progenitor Cell Program, Fox Chase Cancer Center, Philadelphia, Pennsylvania, USA.

\begin{abstract}
The Wnt pathway has been found to play a role in the development of many tissues and to spur growth and differentiation of adult osteoblasts, sparking interest in its potential clinical application for bone growth. However, when deregulated, this pathway can be oncogenic in some tissues. In this issue of the JCI, Kansara and colleagues reveal that Wnt inhibitory factor 1 is epigenetically silenced in human osteosarcomas and that its absence augments osteosarcoma formation in mice (see the related article beginning on page 837). These observations suggest the need for caution in stimulating the Wnt pathway for therapeutic bone growth.
\end{abstract}

Bone loss is a significant clinical concern. It can be caused by aging or by several diseases and their treatments, such as glucocorticoid hormone therapy for autoimmune disease $(1,2)$. Hip fracture in the elderly, an important complication of bone loss, carries a 1-year mortality rate of approximately $25 \%$ $(3,4)$. Treatment and prophylaxis of bone loss has focused on supporting bone mineralization and inhibiting bone resorption, but attention has increasingly turned to building bone by augmenting osteoblast function $(5,6)$. Some drugs under development act in part through stimulating the Wnt signaling pathway, known to drive osteoblast proliferation and commitment $(6,7)$. In particular, efforts are underway to block the actions of secreted Wnt antagonists such as sclerostin (5). The goal of taking advantage of our burgeoning knowledge of signaling pathways in order to build tissue in general and repair bones in particular has great appeal. The theoretical potential exists for the Wnt pathway to serve as a golden goose, generating new bone indefinitely.

Conflict of interest: The author has declared that no conflict of interest exists.

Nonstandard abbreviations used: APC, adenomatous polyposis coli; SFRP, secreted Frizzled-related protein; WIF, Wnt inhibitory factor.

Citation for this article: J. Clin. Invest. 119:758-760 (2009). doi:10.1172/JCI38973.

\section{The Wnt pathway}

The Wnt pathway is a workhorse of development in multicellular organisms. It directs fate decisions, big and small, such as forming a principal axis of frog embryo development (8) or sculpting heart valves (9). Wnt signaling often drives tissue formation. This functionality endures in some adult tissues that require continuous replenishment, such as the renewal of the intestinal epithelium (10).

However, the Wnt pathway is also the prototypical developmental pathway deregulated in cancer (11). In fact, the pathway's name embodies this potential: Wnt is a contraction of Wingless from Drosophila and Int1 from mammals (12). The Wingless gene was discovered as the site of a mutation in Drosophila responsible for defective patterning of the trunk (13). Int1, the first mammalian homolog of Wingless, was discovered as a common site of integration of mouse mammary tumor virus genomes in tumors induced by the virus (14). These insertion events mediate Wnt1 overexpression and tumor growth. The tendency of Wht deregulation to foster neoplasia is a concern.

The canonical Wnt pathway is liberally endowed with regulatory steps (Figure 1). Wnts, the ligands, are low-abundance secreted factors that are somewhat lipophilic $(15,16)$. For a long time, Wnts could not be isolated biochemically, even when overexpressed in cell culture. Expression was inferred from their biological effects (16). These properties likely reflect the role of Wht proteins as short-range paracrine factors, thought to often be present in steep gradients of abundance. Wnt proteins bind 7-pass transmembrane domain receptors of the Frizzled family (11). Such binding is antagonized by the Wnt inhibitory factors (WIFs) and secreted Frizzled-related proteins (SFRPs), which are all secreted proteins that compete with receptors for ligand binding. Ligand binding activates the Frizzled receptors, transducing a signal through the scaffolding protein Dishevelled and Casein kinase 1 to a protein complex that contains the kinase glycogen synthase kinase- 3 and the tumor suppressor proteins adenomatous polyposis coli (APC; named for the human genetic disease also termed familial adenomatous polyposis) and axis inhibition protein 1 . The APC complex constitutively targets $\beta$-catenin for ubiquitination and degradation. The Wnt signal inhibits the APC complex, leading to stabilization of $\beta$-catenin and its accumulation in the nucleus. There, $\beta$-catenin teams with $\mathrm{T}$ cell factor transcription factors to drive expression of genes such as $c-M y c$ and Cyclin D1 that support cell growth, proliferation, and survival. The pivotal role of Wnt signaling in normal and abnormal growth is underscored by the fact that multiple pathway components have been implicated as either oncogenes or tumor suppressors (Figure 1).

\section{WIF1 and osteosarcoma}

In this issue of the JCI, Kansara et al. (17) examined genes that were epigenetically silenced in osteosarcoma with the notion that these may be important tumor sup- 


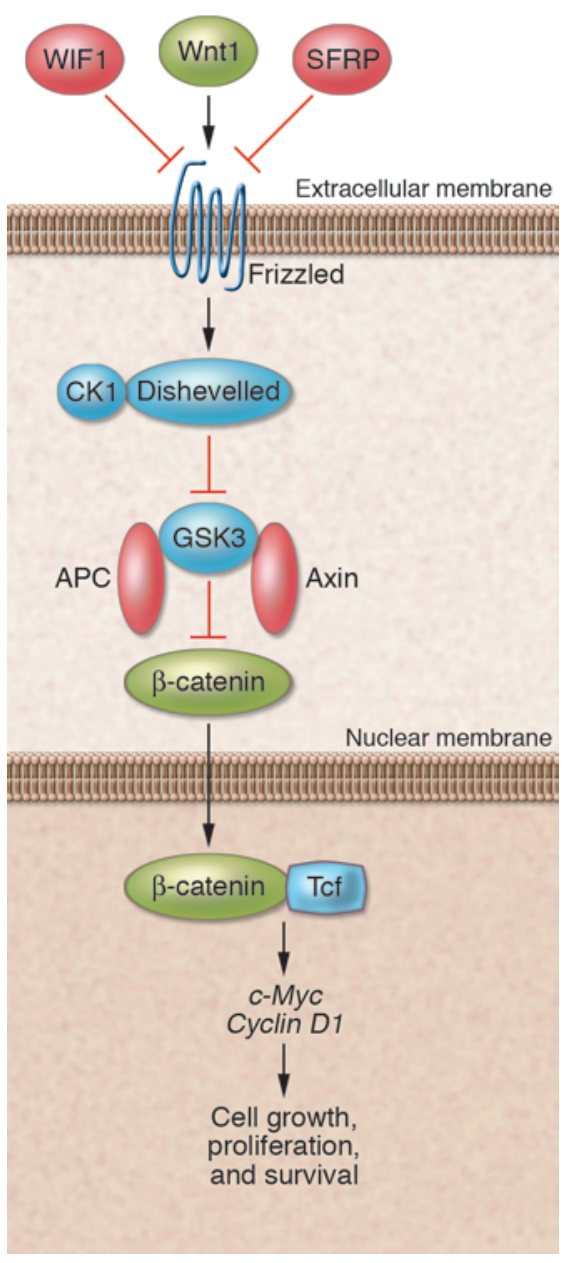

pressors. Although it is known that the Wnt pathway is primarily altered through mutation in tumorigenesis, a large body of evidence now suggests that epigenetic changes are also broadly important in the development of cancer (18), including deregulation of the Wnt pathway (19). Such changes do not alter the DNA sequences of genes, but do alter the structure of chromatin - a complex of DNA and tightly bound proteins that compacts DNA and renders it more or less accessible to transcription factors. There are diverse modes of epigenetic gene regulation. Among the most prominent is DNA methylation. One of the best examples of epigenetic regulation in cancer is methylation-based silencing of the tumor suppressor $\mathrm{p}^{16^{\text {Ink4a }}}(20)$. This suppressor is frequently inactivated by mutations in conditions such as familial melanoma or sporadic pancreatic carcinoma, but is inactivated primarily by methylation in cancers of the colon and other tissues $(18,21)$.

Kansara et al. identified WIF1 among a small set of potential tumor suppressor

\section{Figure 1}

The Wnt signaling pathway. Wnt ligands bind to transmembrane receptors of the Frizzled family. Secreted factors such as WIFs and SFRPs compete with Wnt ligands for binding. Activated receptor signals through Dishevelled and Casein kinase 1 (CK1) to a protein complex containing glycogen synthase kinase-3 (GSK3), axis inhibition protein 1 (Axin), and APC. The APC complex constitutively directs $\beta$-catenin ubiquitination and degradation. The Wnt signal inhibits the APC complex, leading to $\beta$-catenin stabilization and accumulation in the nucleus. Nuclear $\beta$-catenin binds T cell factor (Tcf) transcription factors to drive expression of genes such as $c-M y c$ and Cyclin D1 that support cell growth, proliferation, and survival. Oncogenes are shown in green; tumor suppressor genes are shown in red. In this issue of the $\mathrm{JCl}$, Kansara et al. provide evidence that WIF1 is a tumor suppressor and that epigenetic silencing of WIF1 accelerates osteosarcomagenesis (17).

genes that became expressed (derepressed) after treatment of human osteosarcoma cell lines with a demethylating agent (17). The authors' choice to use genome-wide transcription profiling to identify transcriptional changes in this setting provided an unbiased analysis, but one that typically requires panning through much chaff to locate the wheat. The authors used several criteria to focus on the most interesting genes. They chose WIF1 in part because the Wnt pathway is known to regulate bone formation (7). The authors further studied the relationship between WIF1 and osteosarcoma through the use of Wif1-knockout mice. Mice harboring a targeted deletion of Wif1 maintained largely normal bone growth, but 2 of 13 animals developed spontaneous osteosarcoma (compared with 0 of 30 control mice). To more robustly test the impact of Wif1 on osteosarcoma formation, mice were administered radioactive calcium, a known bone carcinogen. The Wif1-knockout mice demonstrated a moderate but highly statistically significant increase in osteosarcoma. Results of further experiments suggested that WIF1 fosters both increased differentiation and reduced proliferation of human osteosarcoma cells.

\section{Caution advised in Wnt-targeted therapy}

In summary, this study reported by Kansara et al. (17) tells a cautionary story. The concept of pharmacological stimulation of the Wnt pathway had already raised concern, given evidence for a pivotal role of this pathway in colon carcinoma (22). Not only is APC mutated in most colon tumors, but epigenetic silencing of SFRP, an event mechanistically and functionally similar to the silencing of WIF1, appears to also play a role in colon tumorigenesis (19). There is emerging evidence that aberrant activation of the Wnt pathway may stave off cellular senescence in many early neoplasms of the colon, melanocytes, and other tissues, coun- teracting this barrier to tumorigenesis (23). The study by Kansara et al. suggests that, even if a Wnt-targeted drug can be developed that acts relatively selectively on bone, there may be an inherent risk of osteosarcoma (17). Thus, therapy that stimulates the Wnt pathway for bone formation could represent a Trojan horse, an initially welcome gift that subsequently unleashes malignant cells that run amok in the host. In selected patients, the risk of bone loss, with its considerable attendant morbidity and mortality, may outweigh a small increase in risk of osteosarcoma. However, the concern for oncogenic effects of pharmacologic stimulation of the Wnt pathway may linger until more distinct differences between normal and neoplastic cells are uncovered in Wnt signaling or its integration with other regulatory networks that determine cell fate. In patients with bone loss and an elevated risk of malignancy based on family or personal history, use of an agent such as zoledronic acid, which counteracts osteoclast function and may antagonize tumor progression, has appeal (24).

\section{Acknowledgments}

The author thanks Peter Adams and David Weinberg for input. Related research in the author's laboratory is supported by NIH grant R01 DK64758.

Address correspondence to: Greg H. Enders, Department of Medicine, W225, Fox Chase Cancer Center, 333 Cottman Avenue, Philadelphia, Pennsylvania 19111, USA. Phone: (215) 214-3956; Fax: (215) 728-4333; E-mail: greg.enders@fccc.edu.

\footnotetext{
1. Dovio, A., Micossi, I., Bianco, E., and Angeli, A. 2008. Determinants of glucocorticoid action in the bone microenvironment. J. Endocrinol. Invest. 31:7-15.

2. Wang, F.S., Ko, J.Y., Yeh, D.W., Ke, H.C., and Wu, H.L. 2008. Modulation of Dickkopf-1 attenuates glucocorticoid induction of osteoblast apoptosis, adipocytic differentiation, and bone mass loss. Endocrinology. 149:1793-1801.

3. Haleem, S., Lutchman, L., Mayahi, R., Grice, J.E.,
} 
and Parker, M.J. 2008. Mortality following hip fracture: trends and geographical variations over the last 40 years. Injury. 39:1157-1163.

4. Lyles, K.W., et al. 2007. Zoledronic acid and clinical fractures and mortality after hip fracture. $N$. Engl. J. Med. 357:1799-1809.

5. Canalis, E., Giustina, A., and Bilezikian, J.P. 2007. Mechanisms of anabolic therapies for osteoporosis. N. Engl. J. Med. 357:905-916.

6. Krishnan, V., Bryant, H.U., and Macdougald, O.A 2006. Regulation of bone mass by Wnt signaling. J. Clin. Invest. 116:1202-1209.

7. Macsai, C.E., Foster, B.K., and Xian, C.J. 2008. Roles of Wnt signalling in bone growth, remodelling, skeletal disorders and fracture repair. J. Cell. Physiol. 215:578-587

8. Kuhl, M., and Pandur, P. 2008. Dorsal axis duplication as a functional readout for Wnt activity. Methods Mol. Biol. 469:467-476.

9. Hurlstone, A.F., et al. 2003. The Wnt/beta-catenin pathway regulates cardiac valve formation. Nature. 425:633-637

10. Korinek, V., et al. 1998. Depletion of epithelial stem-cell compartments in the small intestine of mice lacking Tcf-4. Nat. Genet. 19:379-383.
11. Logan, C.Y., and Nusse, R. 2004. The Wnt signaling pathway in development and disease. Annu. Rev. Cell Dev. Biol. 20:781-810.

12. Nusse, R., et al. 1991. A new nomenclature for int -1 and related genes: the Wnt gene family. Cell. 64:231.

13. Sharma, R.P., and Chopra, V.L. 1976. Effect of the Wingless (wg1) mutation on wing and haltere development in Drosophila melanogaster. Dev. Biol. 48:461-465.

14. Nusse, R., and Varmus, H.E. 1982. Many tumors induced by the mouse mammary tumor virus contain a provirus integrated in the same region of the host genome. Cell. 31:99-109.

15. Brown, A.M., Papkoff, J., Fung, Y.K., Shackleford, G.M., and Varmus, H.E. 1987. Identification of protein products encoded by the proto-oncogene int-1. Mol. Cell. Biol. 7:3971-3977.

16. Brown, A.M., Wildin, R.S., Prendergast, T.J., and Varmus, H.E. 1986. A retrovirus vector expressing the putative mammary oncogene int- 1 causes partial transformation of a mammary epithelial cell line. Cell. 46:1001-1009.

17. Kansara, M., et al. 2009. Wnt inhibitory factor 1 is epigenetically silenced in human osteosarcoma, and targeted disruption accelerates osteosarcomagenesis in mice. J. Clin. Invest. 119:837-851.

18. Baylin, S.B., Herman, J.G., Graff, J.R., Vertino, P.M., and Issa, J.-P. 1998. Alterations in DNA methylation: a fundamental aspect of neoplasia. Adv. Cancer Res. 72:141-196.

19. Suzuki, H., et al. 2004. Epigenetic inactivation of SFRP genes allows constitutive WNT signaling in colorectal cancer. Nat. Genet. 36:417-422.

20. Herman, J.G., et al. 1995. Inactivation of the CDKN2/p16/MTS1 gene is frequently associated with aberrant DNA methylation in all common human cancers. Cancer Res. 55:4525-4530.

21. Liggett, W.H., Jr., and Sidransky, D. 1998. Role of the p16 tumor suppressor gene in cancer. J. Clin. Oncol. 16:1197-1206.

22. van de Wetering, M., et al. 2002. The beta-catenin/ TCF-4 complex imposes a crypt progenitor phenotype on colorectal cancer cells. Cell. 111:241-250.

23. Adams, P.D., and Enders, G.H. 2008. Wnt signaling and senescence: A tug of war in early neoplasia? Cancer Biol. Ther. 7:1706-1711.

24. Gnant, M., et al. 2009. Endocrine Therapy plus Zoledronic Acid in Premenopausal Breast Cancer. N. Engl.J. Med. 360:679-691.

\title{
Can licorice lick colon cancer?
}

\author{
Paul M. Stewart ${ }^{1}$ and Stephen M. Prescott ${ }^{2}$
}

\begin{abstract}
${ }^{1}$ College of Medical and Dental Sciences, University of Birmingham, Birmingham, United Kingdom. ${ }^{20 k l a h o m a ~ M e d i c a l ~ R e s e a r c h ~ F o u n d a t i o n, ~}$
\end{abstract} Oklahoma City, Oklahoma, USA.

\begin{abstract}
COX-2 promotes colon cancer. While both nonselective NSAIDs and selective COX-2 inhibitors reduce disease burden, their adverse gastrointestinal and cardiovascular side effects limit their therapeutic use. In this issue of the JCI, Zhang et al. used gene silencing and a derivative of licorice root to show that inhibition of the enzyme $11 \beta$-hydroxysteroid dehydrogenase type II (11ßHSD2) reduces tumor COX-2 activity, tumor growth, and metastasis by increasing the tonic glucocorticoid-mediated suppression of the COX-2 signaling pathway without the adverse effects associated with NSAIDs and selective COX-2 inhibitors (see the related article beginning on page 876). Their findings suggest that 11 $\beta$ HSD2 inhibition may be a potential therapeutic option in colon cancer, warranting further investigation.
\end{abstract}

COX-2 is a crucial enzyme in the synthesis of prostaglandins and prostacyclin, which play a variety of roles in the regulation of cell growth, hemostasis, sensing of pain, and inflammation. In normal colon tissue, there is little or no expression of COX-2; however, COX-2 expression is induced early in colon carcinogenesis, is key to disease progression, and influences the clinical course of disease (Figure 1, A and $\mathrm{B}$, and reviewed in ref. 1 ). The COX-2

Conflict of interest: The authors have declared that no conflict of interest exists.

Nonstandard abbreviations used: GE, glycyrrhetinic acid; $11 \beta$ HSD, $11 \beta$-hydroxysteroid dehydrogenase; MR mineralocorticoid receptor.

Citation for this article: J. Clin. Invest. 119:760-763

(2009). doi:10.1172/JCI38936. response clearly plays a central role in colon carcinogenesis, because inhibitors of COX-2 enzymatic activity prevent the development of intestinal polyps in mice and humans (reviewed in ref. 1), and deletion of Cox2 in mice almost completely protects the animals from the development of these polyps (reviewed in ref. 1). However, enthusiasm for the prevention of colon cancer via pharmacological COX-2 inhibition has been tempered by the recognition that such a prevention strategy inherently requires long-term exposure to COX-2 inhibitors. Unfortunately, traditional NSAIDs, which are nonselective COX inhibitors, can cause gastrointestinal hemorrhage, among other complications (2), while selective COX-2 inhibitors confer an increased risk of cardiovascular death (3). Thus, a detailed understanding of how COX-2 expression is induced would be potentially valuable from two perspectives - it would provide insight into both the molecular steps involved in carcinogenesis and potential therapeutic targets.

That COX-2 is overexpressed in colon polyps and cancer has been recognized for more than 15 years (reviewed in ref. 1), but the molecular basis for this overexpression has remained unclear despite extensive investigation of the regulation of the COX2 gene in many experimental settings. It is likely that what was originally thought to be a cell-autonomous event is instead a response to extracellular signals - a "field effect," with growth factors providing much of the signal that results in induction of COX2. From the time of the discovery of COX2 as an early inducible gene, it was almost immediately recognized that COX2 induction in vitro could be inhibited by a class of steroid hormones known as glucocorticoids $(4,5)$. This pharmacologic effect has been attributed to changes in both COX-2 transcription and mRNA stability (6). However, it was not known whether COX-2 was regulated by endogenous glucocorticoids, the most important of which 